\title{
Directly Quantifiable Biotinylation Using a Water-Soluble Isatoic Anhydride Platform
}

Adam B. Fessler, Anthony J. Fowler, Craig A. Ogle*

${ }^{1}$ Department of Chemistry, University of North Carolina at Charlotte, Charlotte, NC 28223.

\section{Table of Contents}

Instrumentation and chemicals

Preparation and characterization of $2 b-2 d$

Preparation and characterization of NHS biotin, 3a-3d

Preparation and characterization of $4 a-4 d$

Degree of Labeling Calculations

S9-S10

Degree of Labeling and HABA comparison.

HRP Labeling and HABA determination

S11-S14

Sulfo-NHS-biotin vs 4a labeling comparison.

S14-S15 


\section{Instrumentation.}

NMR data was obtained on a JEOL ECA 500 NMR. High resolution MS data were analyzed on an Thermo Scientific Orbitrap XL in positive mode at a resolution of 60000, spray voltage of 5 $\mathrm{kV}$, sheath 12, Aux 10, Sweep 0, Capillary Temp $275^{\circ} \mathrm{C}$, Capillary Voltage $32.00 \mathrm{~V}$, Tube Lens of $85.00 \mathrm{~V}$. Compounds were dissolved in $1 \mathrm{~mL}$ acetonitrile (ACN), then $8 \mu \mathrm{L}$ was diluted in 1:1 H2O:ACN with $0.1 \%$ Formic acid. $2 \mu \mathrm{L}$ of this solution was injected using an Accela $1250 \mathrm{LC}$ system, at a solvent flowrate of $200 \mu \mathrm{L} / \mathrm{min}$ of $1: 1 \mathrm{H}_{2} \mathrm{O}$ :ACN with $0.1 \%$ Formic Acid. UV-VIS and Fluorescence data were obtained using a Molecular Devices Spectramax M5 plate reader.

Chemicals. All chemicals were purchased commercially and used without further purification unless otherwise stated.

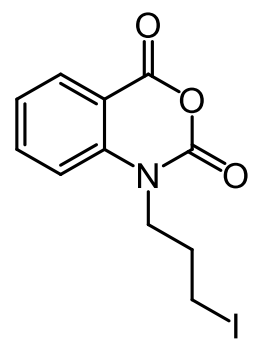

1. This reagent was prepared as previously detailed. ${ }^{1}$

General Procedure 1. To a round bottom flask flushed with $\mathrm{N}_{2}$ was added 3-dimethylamino-1propanol $(5.0 \mathrm{~g}, 48.5 \mathrm{mmol})$. To this was added 0.8 eq $\mathrm{N}, \mathrm{N}$ '-carbonyldiimidazole $(6.3 \mathrm{~g}, 38.8$ mmol) in dry $\mathrm{ACN}$ (40 mL). This solution was stirred for 1 hour then was taken into a syringe. The solution was slowly added to 10 eq. of a diamine $\left(388 \mathrm{mmol}\right.$ ) vigorously stirring under $\mathrm{N}_{2}$. This mixture is stirred for 4 hours then concentrated under reduced pressure. The resulting oil is purified by bulb to bulb distillation for 4 hours are $120{ }^{\circ} \mathrm{C}$, removing excess diamine and 
imidazole, leaving the product in the round bottom. The purified liquid is allowed to cool prior to removal from the distillation as to avoid charring of the sample.

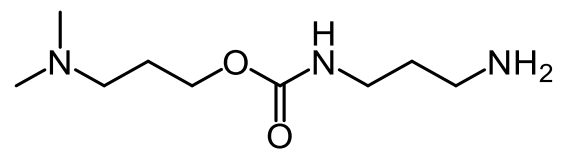

2b. General procedure 1 using 1,3-propane diamine was followed for the preparation of this reagent. Final product was collected as a yellow oil. (5.52g, 70\%) $\mathrm{C}_{9} \mathrm{H}_{21} \mathrm{~N}_{3} \mathrm{O}_{2}(203.29){ }^{1} \mathrm{H}$ NMR (500 MHz, DMSO-d 6$): \delta 7.03(\mathrm{t}, 1 \mathrm{H}), 3.90(\mathrm{t}, 2 \mathrm{H}), 2.97(\mathrm{q}, 2 \mathrm{H}), 2.48(\mathrm{t}, 2 \mathrm{H}), 2.19(\mathrm{t}, 2 \mathrm{H}), 2.07(\mathrm{~s}$, 6H), $1.61(\mathrm{p}, 2 \mathrm{H}), 1.40(\mathrm{p}, 2 \mathrm{H}) \mathrm{ppm},{ }^{13} \mathrm{C}$ NMR (125 MHz, DMSO-d 6$): \delta 156.9,62.6,56.2,45.7$, 39.9, 38.5, 33.9 ppm. HRMS (ESI): Calc. 204.1712; Found: $204.1706\left(\mathrm{M}^{+}+\mathrm{H}\right) \mathrm{m} / \mathrm{z}$.

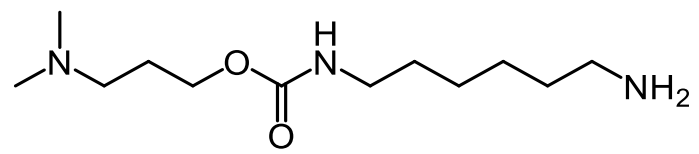

2c. General procedure 1 using 1,6-hexamethylene diamine was followed for the preparation of this reagent. Final product was collected as an off-white solid. (6.66g, 70\%) $\mathrm{C}_{12} \mathrm{H}_{27} \mathrm{~N}_{3} \mathrm{O}_{2}(245.37){ }^{1} \mathrm{H}$ NMR (500 MHz, DMSO-d $)$ ): $\delta 7.02$ (t, 1H), 3.89 (t, 2H), 2.90 (q, 2H), 2.47 (t, 2H), $2.46(t, 2 \mathrm{H})$, $2.19(\mathrm{t}, 2 \mathrm{H}), 2.07(\mathrm{~s}, 6 \mathrm{H}), 1.61(\mathrm{p}, 2 \mathrm{H}), 1.33(\mathrm{~m}, 2 \mathrm{H}), 1.28(\mathrm{~m}, 2 \mathrm{H}), 1.20(\mathrm{~m}, 4 \mathrm{H}) \mathrm{ppm},{ }^{13} \mathrm{C} \mathrm{NMR}$ (125 MHz, DMSO-d 6 ): $\delta$ 156.2, 61.9, 55.6, 45.1, 41.5, 40.0, 33.3, 29.4, 26.9, 26.1, 26.0 ppm. HRMS (ESI): Calc. 246.2182; Found: $246.2176\left(\mathrm{M}^{+}+\mathrm{H}\right) \mathrm{m} / \mathrm{z}$.<smiles>CN(C)CCCOC(=O)NCCCOCCOCCOCCCN</smiles>

$\underline{\mathbf{2 d}}$. General procedure 1 using 4,7,10-trioxa-1,13-tridecanediamine was followed with the following modifications for the preparation of this reagent. Reaction was scaled down to $5 \mathrm{mmol}$ CDI starting material and bulb to bulb distillation was performed for $6 \mathrm{~h}$ to remove excess diamine. Final product was isolated as a yellow oil $(0.87 \mathrm{~g}, 50 \%) . \mathrm{C}_{16} \mathrm{H}_{35} \mathrm{~N}_{3} \mathrm{O}_{5}$ (349.47), ${ }^{1} \mathrm{H}$ NMR (500 
MHz, DMSO-d $\left.{ }_{6}\right): \delta 4.15(\mathrm{t}, 2 \mathrm{H}), 3.30(\mathrm{~m}, 16 \mathrm{H}), 2.58(\mathrm{t}, 2 \mathrm{H}), 2.12(\mathrm{t}, 2 \mathrm{H}), 1.96(\mathrm{~s}, 6 \mathrm{H}), 1.65(\mathrm{p}$, 2H), 1.56 (p, 2H), 1.48 (p, 2H) ppm, ${ }^{13} \mathrm{C}$ NMR (125 MHz, DMSO-d 6 ): $\delta$ 156.3, 69.78, 69.76, 69.53, 69.49, 68.5, 68.0, 62.0, 55.6, 45.1, 38.8, 37.5, 33.3, 29.6, 26.9. HRMS (ESI): Calc. 3350.2655; Found: $350.2649\left(\mathrm{M}^{+}+\mathrm{H}\right) \mathrm{m} / \mathrm{z}$.

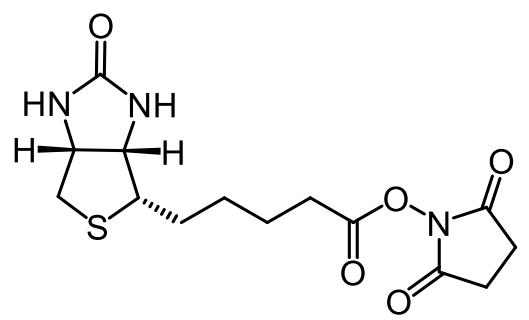

NHS-Biotin. To a round bottom containing biotin ( $3 \mathrm{~g}, 12.3 \mathrm{mmol})$ stirring in dry DMF (100 mL) under $\mathrm{N}_{2}$, was added 1.2 eq 1-ethyl-3-(3-dimethylaminopropyl)carbodiimide (2.8g, $\left.14.7 \mathrm{mmol}\right)$. To this solution was added 1.25 eq $\mathrm{N}$-hydroxysuccinimide $(1.8 \mathrm{~g}, 15.5 \mathrm{mmol})$. This solution was stirred overnight then poured into a $1000 \mathrm{~mL}$ beaker containing ice $(500 \mathrm{~g})$ resulting in the precipitation of a white solid. Solid is collected through filtration over glass frit then washed with chilled methanol. Solid is collected and placed under vacuum overnight. Final product was collected as a white powder $(3.35 \mathrm{~g}, 80 \%) . \mathrm{C}_{14} \mathrm{H}_{19} \mathrm{~N}_{3} \mathrm{O}_{5} \mathrm{~S}$ (341.38), ${ }^{1} \mathrm{H}$ NMR (500 MHz, DMSO$\left.\mathrm{d}_{6}\right): \delta 6.41(\mathrm{~s}, 1 \mathrm{H}), 6.35(\mathrm{~s}, 1 \mathrm{H}), 4.27(\mathrm{t}, 1 \mathrm{H}), 4.11(\mathrm{t}, 1 \mathrm{H}), 3.07(\mathrm{q}, 1 \mathrm{H}), 2.80(\mathrm{t}, 1 \mathrm{H}), 2.78(\mathrm{~s}, 4 \mathrm{H})$ $2.64(\mathrm{t}, 2 \mathrm{H}) 2.54(\mathrm{~s}, 1 \mathrm{H}), 1.45(\mathrm{~m}, 6 \mathrm{H}) \mathrm{ppm},{ }^{13} \mathrm{C}$ NMR (125 MHz, DMSO-d 6$): \delta 170.3,168.9$, $162.7,60.9,59.1,55.2,40.8,29.9,27.8,27.5,25.4,24.3$ ppm. Compared to published spectra. ${ }^{2}$

General Procedure 2. To a round bottom flask under $\mathrm{N}_{2}$ was added 3 eq. (3.6 mmol) $\alpha-\mathrm{N}, \mathrm{N}-$ dimethyl- $\Omega$-diamine (2a-2d) in $20 \mathrm{~mL}$ dry DMF. To this was added a suspension of $\mathbf{3}$ in $20 \mathrm{~mL}$ dry DMF $(0.4 \mathrm{~g}, 1.2 \mathrm{mmol})$. The suspension was stirred vigorously at $30^{\circ} \mathrm{C}$ until the cloudy solution went clear, turning pale yellow. The reaction was stirred at RT for $8 \mathrm{~h}$ then concentrated under reduced vacuum. Product was purified via column chromatography over flash silica 70-

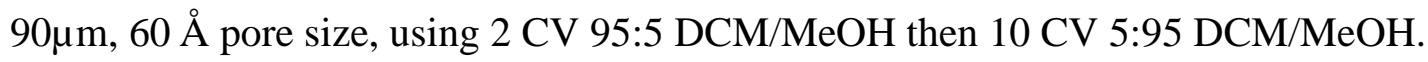




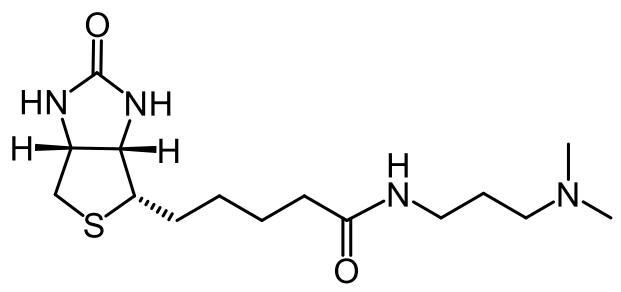

3a. General procedure 2 using 2a was followed for the preparation of this reagent. Product was isolated as a pale yellow solid (323 mg, 82\%). $\mathrm{C}_{15} \mathrm{H}_{28} \mathrm{~N}_{4} \mathrm{O}_{2} \mathrm{~S}(328.48){ }^{1} \mathrm{H}$ NMR (500 MHz, DMSO$\left.\mathrm{d}_{6}\right): \delta 7.75(\mathrm{t}, 1 \mathrm{H}), 6.42(\mathrm{~s}, 1 \mathrm{H}), 6.35(\mathrm{~s}, 1 \mathrm{H}), 4.30(\mathrm{t}, 1 \mathrm{H}), 4.12(\mathrm{~m}, 1 \mathrm{H}), 3.09(\mathrm{~m}, 1 \mathrm{H}), 3.03(\mathrm{~d}$, 2H), $2.82(\mathrm{dd}, 1 \mathrm{H}), 2.87(\mathrm{t}, 4 \mathrm{H}), 2.18(\mathrm{t}, 2 \mathrm{H}), 2.11(\mathrm{~s}, 6 \mathrm{H}), 2.04(\mathrm{t}, 2 \mathrm{H}), 1.45(\mathrm{~m}, 8 \mathrm{H}) \mathrm{ppm},{ }^{13} \mathrm{C}$ NMR (125 MHz, DMSO-d 6 ): $\delta 173.2,172.1,163.0,61.3,59.5,56.9,55.8,45.4,37.0,35.5,28.5$, 28.4, 27.5, 25.5 ppm. HRMS (ESI): Calc. 329.2011; Found: $329.2006\left(\mathrm{M}^{+}+\mathrm{H}\right) \mathrm{m} / \mathrm{z}$.

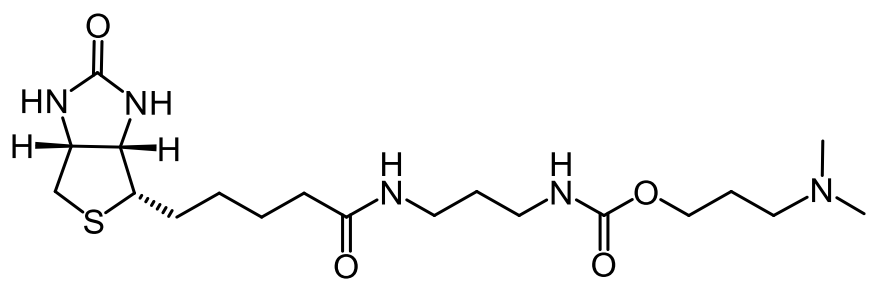

$\underline{\mathbf{3 b}}$. General procedure 2 using $\mathbf{2 b}$ was followed for the preparation of this reagent. Final product was isolated as a pale yellow solid (309 mg, 60\%). $\mathrm{C}_{19} \mathrm{H}_{35} \mathrm{~N}_{5} \mathrm{O}_{4} \mathrm{~S}(429.58),{ }^{1} \mathrm{H} \mathrm{NMR}(500 \mathrm{MHz}$, DMSO-d $\left.\mathrm{d}_{6}\right): \delta 7.743(\mathrm{t}, 1 \mathrm{H}), 7.02(\mathrm{t}, 1 \mathrm{H}), 6.41(\mathrm{~s}, 1 \mathrm{H}), 6.34(\mathrm{~s}, 1 \mathrm{H}), 4.30(\mathrm{t}, 1 \mathrm{H}) 4.12(\mathrm{t}, 1 \mathrm{H}) 3.94$ $(\mathrm{t}, 2 \mathrm{H}), 3.09(\mathrm{~m}, 1 \mathrm{H}) 3.02(\mathrm{q}, 2 \mathrm{H}), 2.95,(\mathrm{q}, 2 \mathrm{H}), 2.82(\mathrm{dd}, 1 \mathrm{H}), 2.57(\mathrm{~d}, 1 \mathrm{H}), 2.23(\mathrm{t}, 2 \mathrm{H}), 2.10(\mathrm{~s}$, 6H), $2.04(\mathrm{t}, 2 \mathrm{H}) 1.35(\mathrm{~m}, 12 \mathrm{H}) \mathrm{ppm},{ }^{13} \mathrm{C}$ NMR (125 MHz, DMSO-d 6 ): $\delta$ 172.2, 162.9, 156.5, 62.4, 61.3, 59.5, 55.9, 55.7, 45.4, 38.3, 36.3, 35.5, 29.8, 28.5, 28.3, 27.2, 25.6 ppm, HRMS (ESI): Calc. 430.2488; Found: $430.2481\left(\mathrm{M}^{+}+\mathrm{H}\right) \mathrm{m} / \mathrm{z}$. 


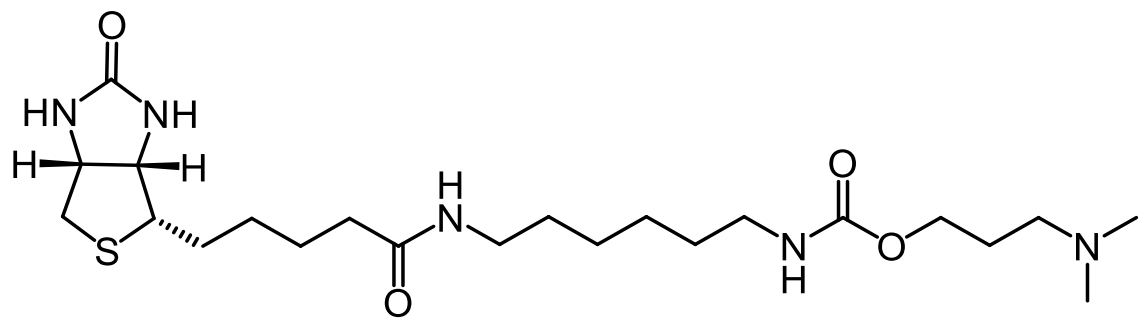

3c. General procedure 2 using $\mathbf{2 c}$ was followed for the preparation of this reagent. Product was isolated as a pale yellow solid (339 mg, 60\%). $\mathrm{C}_{22} \mathrm{H}_{41} \mathrm{~N}_{5} \mathrm{O}_{4} \mathrm{~S}$ (471.66) ${ }^{1} \mathrm{H}$ NMR (500 MHz, DMSO$\left.\mathrm{d}_{6}\right): \delta 7.71(\mathrm{t}, 1 \mathrm{H}), 7.03(\mathrm{t}, 1 \mathrm{H}), 6.42(\mathrm{~s}, 1 \mathrm{H}), 6.35(\mathrm{~s}, 1 \mathrm{H}), 4.30(\mathrm{t}, 2 \mathrm{H}), 4.12(\mathrm{t}, 1 \mathrm{H}), 3.93(\mathrm{t}, 2 \mathrm{H})$. $3.09(\mathrm{~m}, 1 \mathrm{H}), 3.00(\mathrm{q}, 2 \mathrm{H}), 2.93(\mathrm{q}, 2 \mathrm{H}), 2.82(\mathrm{~d}, 1 \mathrm{H}), 2.57(\mathrm{~d}, 1 \mathrm{H}), 2.22(\mathrm{t}, 2 \mathrm{H}), 2.10(\mathrm{~s}, 6 \mathrm{H}), 2.04$ (t, 2H), ${ }^{13} \mathrm{C}$ NMR (125 MHz, DMSO-d 6 ): $\delta$ 172.2, 163.2, 156.9, 62.4, 61.5, 59.6, 56.1, 55.9, 45.6, 38.8, 35.7, 29.9, 29.6, 28.7, 28.5. 27.4, 26.5, 26.4, 25.8 ppm, HRMS (ESI): Calc. 472.2958; Found: $472.2951\left(\mathrm{M}^{+}+\mathrm{H}\right) \mathrm{m} / \mathrm{z}$.

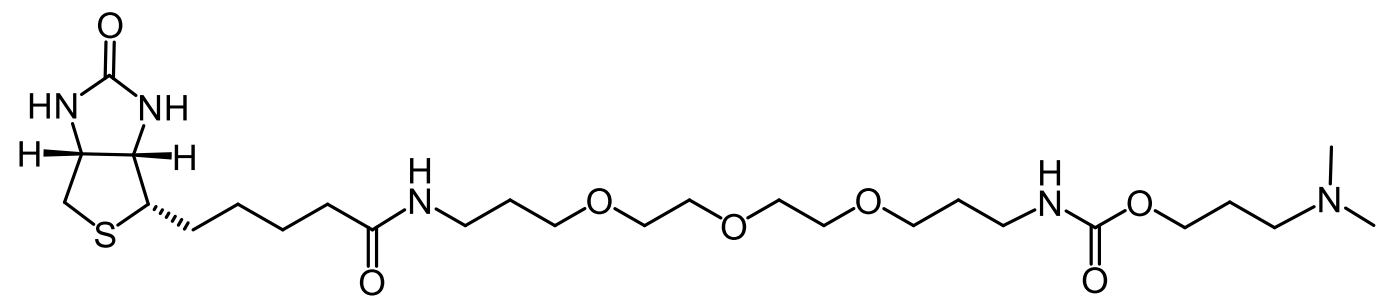

3d. General procedure 2 using $\mathbf{2 d}$ was followed for the preparation of this reagent with the following modification, $2.2 \mathrm{mmol} \mathbf{2 d}$ and $0.7 \mathrm{mmol}$ 3. Product was isolated as a yellow oil. (200 $\mathrm{mg}, 50 \%) \mathrm{C}_{26} \mathrm{H}_{49} \mathrm{~N}_{5} \mathrm{O}_{7} \mathrm{~S}$ (575.77), ${ }^{1} \mathrm{H}$ NMR (500 Mz, MeOD): $\delta 4.99$ (t, 1H), 4.299 (q, 1H), 4.10 (t, 2H), $3.51(\mathrm{~m}, 6 \mathrm{H}), 3.19(\mathrm{~m}, 8 \mathrm{H}), 2.92(\mathrm{q}, 1 \mathrm{H}), 2.81,(\mathrm{~s}, 6 \mathrm{H}), 2.70(\mathrm{~d}, 1 \mathrm{H}), 2.19(\mathrm{t}, 2 \mathrm{H}), 1.75(\mathrm{~m}$, 8H), $1.42(\mathrm{p}, 2 \mathrm{H}), 1.30(\mathrm{t}, 4 \mathrm{H}), 1.16$ (t, 2H), ${ }^{13} \mathrm{C}$ NMR (125 MHz, MeOD): $\delta$ 175.8, 165.9, 158.5, $71.5,71.2,69.9,69.7,63.3,62.6,61.6,58.3,56.9,56.5,47.8,43.8,41.1,39.1,37.8,36.8,30.8$ 30.4, 29.8, 26.3 18.4, 9.3 ppm. HRMS (ESI): Calc. 576.3431; Found: $576.3423\left(\mathrm{M}^{+}+\mathrm{H}, 100 \%\right)$ $\mathrm{m} / \mathrm{z}$. 
General Procedure 3. To a $1.5 \mathrm{~mL}$ vial flushed with $\mathrm{N}_{2}$ was added $\mathbf{1}(100 \mathrm{mg}, 0.3 \mathrm{mmol})$ in 750 $\mu \mathrm{L}$ dry acetone through a $0.2 \mu \mathrm{m}$ filter. To this was added 0.9 eq of biotin derivative (3a-3d) $(0.27$ mmol) in $750 \mu \mathrm{L}$ dry DMF. The vial is sealed and placed at $40^{\circ} \mathrm{C}$ for $24 \mathrm{hrs}$; the solution was transferred to a dry centrifuge vial. To this was added diethyl ether until the solution became turbid ( $\sim 5 \mathrm{~mL}$ ), the solution was mixed thoroughly until clear. To this additional ethyl ether was added quickly ( $\sim 5 \mathrm{~mL})$, vortexed vigorously then centrifuged to collect precipitated solid. The solution was carefully decanted and the oily solid was washed $3 \mathrm{X}$ with ethyl ether, decanted and placed in vacuo for 48 hours.

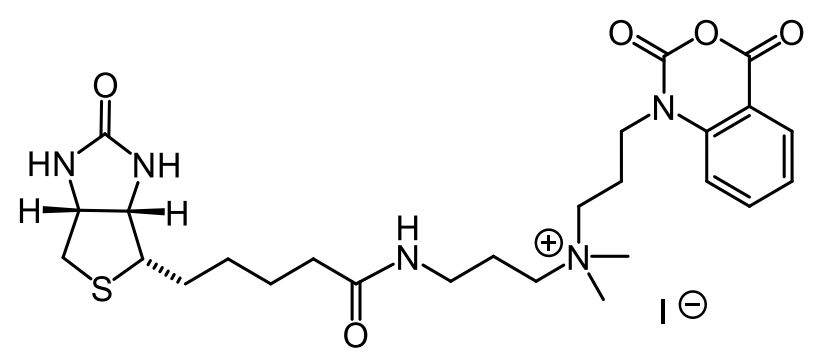

4a. General procedure 3 using 3a was followed for the preparation of this reagent. Product was isolated as a pale yellow solid. (115 mg, 65\%) $\mathrm{C}_{26} \mathrm{H}_{38} \mathrm{IN}_{5} \mathrm{O}_{5} \mathrm{~S}(659.58),{ }^{1} \mathrm{H}$ NMR (500 Mz, DMSO$\left.\mathrm{d}_{6}\right): \delta 8.06(\mathrm{~d}, 1 \mathrm{H}), 7.89(\mathrm{t}, 1 \mathrm{H}), 7.53(\mathrm{~d}, 1 \mathrm{H}), 7.30(\mathrm{t}, 1 \mathrm{H}), 6.39(\mathrm{~s}, 1 \mathrm{H}), 6.36(\mathrm{~s}, 1 \mathrm{H}), 4.31(\mathrm{t}, 1 \mathrm{H})$, $4.12(\mathrm{~m}, 2 \mathrm{H}), 3.43(\mathrm{~m}, 2 \mathrm{H}), 3.22(\mathrm{~m}, 2 \mathrm{H}), 3.11(\mathrm{~m}, 3 \mathrm{H}), 2.97(\mathrm{~s}, 6 \mathrm{H}), 2.81(\mathrm{~d}, 1 \mathrm{H}), 2.57(\mathrm{t}, 2 \mathrm{H})$ $2.06(\mathrm{~m}, 4 \mathrm{H}), 1.80(\mathrm{~m}, 2 \mathrm{H}), 1.60(\mathrm{~m}, 2 \mathrm{H}), 1.50(\mathrm{~m}, 3 \mathrm{H}), 1.30(\mathrm{~m}, 2 \mathrm{H}) \mathrm{ppm},{ }^{13} \mathrm{C} \mathrm{NMR}(125 \mathrm{MHz}$ DMSO-d $\left.{ }_{6}\right): \delta 173.3,162.7,159.8,137.6,130.2,124.2,114.5,62.9,61.6,61.4,59.8,55.4,50.7$, 42.8, 41.6, 40.2, 35.6, 35.2, 28.0, 25.2, 23.0, 20.7 ppm, HRMS (ESI) Calc. 532.2594; Found: $532.2592\left(\mathrm{M}^{+}\right) \mathrm{m} / \mathrm{z}$. 


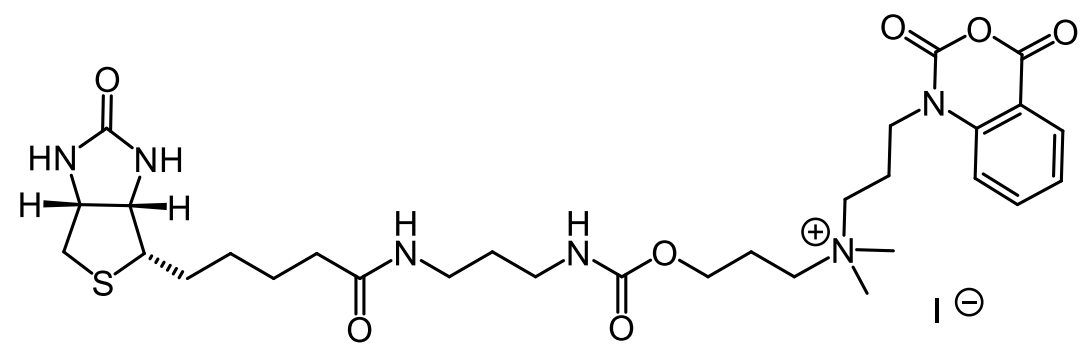

$\underline{4 \mathbf{b}}$. General procedure 3 using $\mathbf{3 b}$ was followed for the preparation of this reagent. Product was isolated as a pale yellow solid. (143 mg, 70\%) $\mathrm{C}_{30} \mathrm{H}_{45} \mathrm{IN}_{6} \mathrm{O}_{7} \mathrm{~S}$ (760.68), ${ }^{1} \mathrm{H}$ NMR (500 Mz, DMSO$\left.\mathrm{d}_{6}\right): \delta 8.03(\mathrm{~d}, 1 \mathrm{H}), 7.85(\mathrm{t}, 1 \mathrm{H}) 7.72(\mathrm{t}, 1 \mathrm{H}), 7.51(\mathrm{~d}, 1 \mathrm{H}), 7.34(\mathrm{~d}, 1 \mathrm{H}), 7.02(\mathrm{t}, 1 \mathrm{H}), 6.36(\mathrm{~s}, 1 \mathrm{H})$, $6.32(\mathrm{~s}, 1 \mathrm{H}), 4.27(\mathrm{t}, 1 \mathrm{H}), 4.08(\mathrm{t}, 3 \mathrm{H}), 3.95(\mathrm{t}, 2 \mathrm{H}), 3.42(\mathrm{~m}, 2 \mathrm{H}), 2.97(\mathrm{~m}, 12 \mathrm{H}), 2.78(\mathrm{~d}, 2 \mathrm{H})$, $2.06(\mathrm{~m}, 2 \mathrm{H}), 2.01(\mathrm{t}, 2 \mathrm{H}), 1.95(\mathrm{~m}, 2 \mathrm{H}), 1.57(\mathrm{~m}, 2 \mathrm{H}), 1.48(\mathrm{~m}, 5 \mathrm{H}), 1.25(\mathrm{~m}, 2 \mathrm{H}) \mathrm{ppm},{ }^{13} \mathrm{C} \mathrm{NMR}$ (125 MHz, DMSO-d6): $\delta 172.3,162.9,159.2,156.0,148.4,141.4,137.5,130.0,124.1,114.9$, $112.1,61.2,61.1,60.9,60.7,59.5,55.6,50.4,41.6,36.3,35.3,34.6,29.8,28.3,25.6,22.3,20.5$ ppm, HRMS (ESI) Calc. 633.3070; Found: $633.3072\left(\mathrm{M}^{+}\right) \mathrm{m} / \mathrm{z}$.

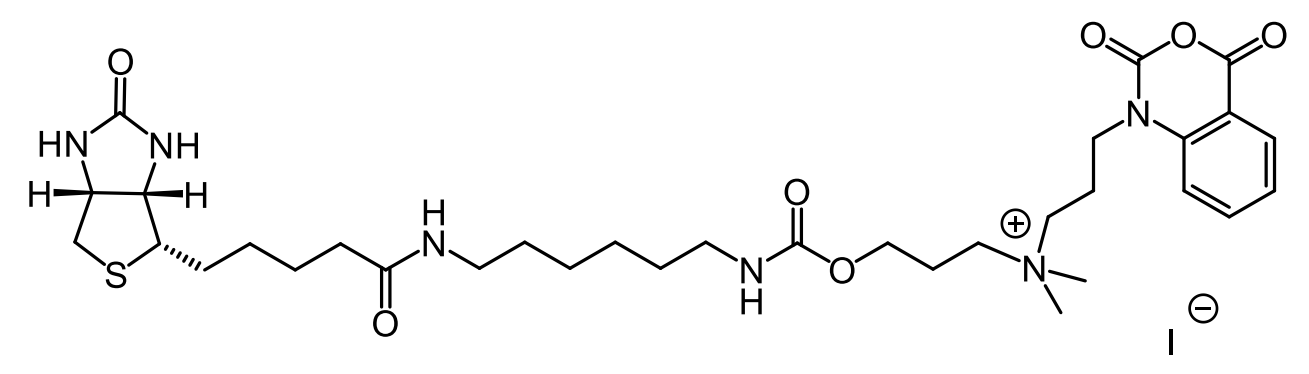

4c. General procedure 3 using $3 \mathbf{c}$ was followed for the preparation of this reagent. Product was isolated as a pale yellow solid. (140 mg, 65\%) $\mathrm{C}_{33} \mathrm{H}_{51} \mathrm{IN}_{6} \mathrm{O}_{7} \mathrm{~S}$ (802.76), ${ }^{1} \mathrm{H}$ NMR (500 MHz, DMSO-d $\left.)_{6}\right): 8.06(\mathrm{~d}, 1 \mathrm{H}), 7.88(\mathrm{t}, 1 \mathrm{H}), 7.71(\mathrm{t}, 1 \mathrm{H}), 7.54(\mathrm{~d}, 1 \mathrm{H}), 7.37(\mathrm{t}, 1 \mathrm{H}), 7.02(\mathrm{t}, 1 \mathrm{H}), 6.40(\mathrm{~s}$, 1H), $6.35(\mathrm{~s}, 1 \mathrm{H}), 4.30(\mathrm{t}, 1 \mathrm{H}), 4.11(\mathrm{t}, 2 \mathrm{H}), 3.97(\mathrm{t}, 2 \mathrm{H}), 3.46(\mathrm{~m}, 2 \mathrm{H}), 3.08(\mathrm{~m}, 2 \mathrm{H}), 3.00(\mathrm{~s}, 8 \mathrm{H})$, $2.94(\mathrm{q}, 2 \mathrm{H}), 2.80(\mathrm{t}, 2 \mathrm{H}), 2.09(\mathrm{~m}, 2 \mathrm{H}), 2.03(\mathrm{t}, 2 \mathrm{H}), 1.98(\mathrm{~m}, 2 \mathrm{H}), 1.60(\mathrm{~m}, 1 \mathrm{H}), 1.49(\mathrm{~m}, 3 \mathrm{H})$, $1.25(\mathrm{~m}, 12 \mathrm{H}) \mathrm{ppm},{ }^{13} \mathrm{C}$ NMR (125 MHz, DMSO-d 6 ): 171.9, 162.9, 159.1, 156.0, 148.0141 .2$, $137.4,129.9,123.9,114.8,112.0,61.2,61.03,61.0,60.6,59.3,55.6,50.3,41.5,38.4,35.4,34.6$, 
29.5, 29.2, 28.4, 28.2, 26.3, 26.0, 25.5, 22.5, 20.4 ppm. HRMS (ESI) Calc. 675.3534; Found: $675.3526\left(\mathrm{M}^{+}\right) \mathrm{m} / \mathrm{z}$.

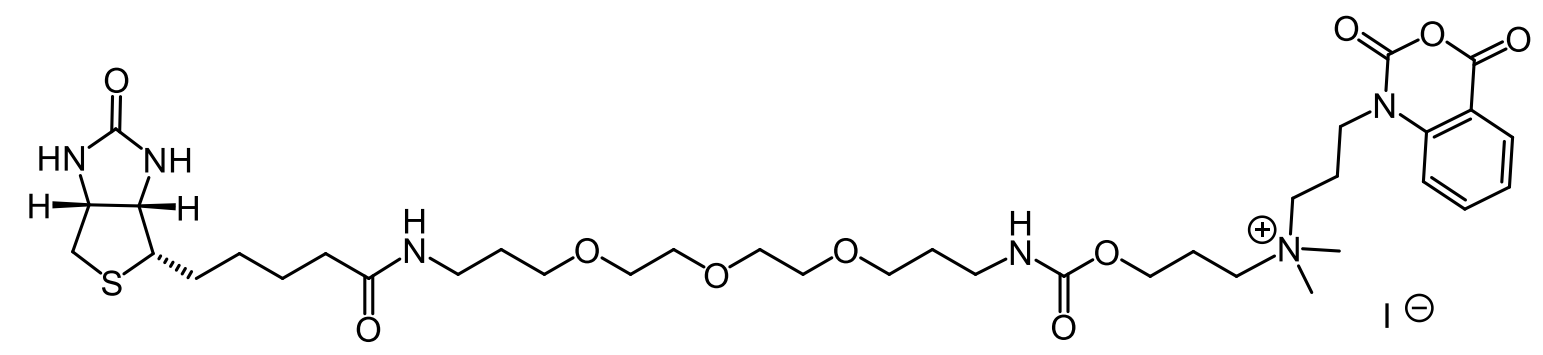

4d. General procedure 3 using $\mathbf{3 d}$ was followed for the preparation of this reagent. Product was isolated as a dark yellow oil. (122 mg, 50\%) $\mathrm{C}_{37} \mathrm{H}_{59} \mathrm{IN}_{6} \mathrm{O}_{10} \mathrm{~S}$ (906.87), ${ }^{1} \mathrm{H}$ NMR (500 Mz, DMSO$\left.\mathrm{d}_{6}\right): \delta 8.07(\mathrm{~d}, 1 \mathrm{H}), 7.88(\mathrm{t}, 1 \mathrm{H}), 7.73(\mathrm{t}, 1 \mathrm{H}), 7.54(\mathrm{t}, 1 \mathrm{H}), 7.37(\mathrm{t}, 1 \mathrm{H}), 7.08(\mathrm{t}, 1 \mathrm{H}), 6.40(\mathrm{~s}, 1 \mathrm{H})$, $6.35(\mathrm{~s}, 1 \mathrm{H}), 4.30(\mathrm{t}, 1 \mathrm{H}), 4.12(\mathrm{t}, 3 \mathrm{H}), 3.98(\mathrm{t}, 2 \mathrm{H}), 3.51(\mathrm{~m}, 5 \mathrm{H}), 3.47(\mathrm{~m}, 6 \mathrm{H}), 3.38(\mathrm{~m}, 5 \mathrm{H}), 3.06$ (q, 4H), $3.00(\mathrm{~s}, 6 \mathrm{H}), 2.55(\mathrm{~m}, 4 \mathrm{H}), 2.04(\mathrm{~m}, 6 \mathrm{H}), 1.61(\mathrm{~m}, 5 \mathrm{H}), 1.61(\mathrm{~m}, 5 \mathrm{H}), 1.48(\mathrm{~m}, 3 \mathrm{H}), 1.29$ (m, 2H) ppm, ${ }^{13} \mathrm{C}$ NMR (125 MHz, DMSO-d 6 ): 175.6, 165.7, 159.2, 158.7, 148.2, 141.4, 137.5, 130.0, 124.1, 114.8, 112.0, 71.5, 71.2, 70.1, 69.8, 64.4, 63.4, 62.6, 61.6, 61.4, 61.1, 58.5, 56.9, 56.5, 50.4, 47.9, 41.1, 39.3, 37.9, 36.7, 30.6, 30.4, 29.9, 26.3, 18.4, 9.3 ppm. HRMS (ESI) Calc. 779.4013; Found: $779.4001\left(\mathrm{M}^{+}\right) \mathrm{m} / \mathrm{z}$.

\section{Degree of Labeling Calculations:}

$$
D O L=\frac{A_{330} \varepsilon_{280 \text { protein }}}{\left(A_{280}-A_{330} C_{280}\right) \varepsilon_{330 \text { reagent }}}
$$

Equation S1: degree of labeling formula

\begin{tabular}{|l|l|}
\hline Abbreviation & Definition \\
\hline $\mathrm{A}_{330}$ & Absorbance of the sample at $330 \mathrm{~nm}$ \\
\hline$\varepsilon_{280 \text { protein }}$ & Molar extinction coefficient $\left(\right.$ in $\left.\mathrm{M}^{-1} \mathrm{~cm}^{-1}\right)$ of pure protein at $280 \mathrm{~nm}$ \\
\hline $\mathrm{A}_{280}$ & Absorbance of the sample at $280 \mathrm{~nm}$ \\
\hline $\mathrm{C}_{280}$ & Correction factor of reagent's contribution to absorbance at $280 \mathrm{~nm}$ \\
\hline$\varepsilon_{330 \text { reagent }}$ & Molar extinction coefficient (in $\mathrm{M}^{-1} \mathrm{~cm}^{-1}$ ) of the reagent at $330 \mathrm{~nm}$ \\
\hline
\end{tabular}

Table S1: Equation S1 variables 
This is an example calculation of a single replicate of low DOL for 4a-BSA sample in Figure 3 using equation $\mathrm{S} 1$.

\begin{tabular}{|l|l|}
\hline Abbreviation & Definition \\
\hline $\mathrm{A}_{330}$ & 0.4711 \\
\hline$\varepsilon_{280 \text { protein }}$ & $43284 \mathrm{M}^{-1} \mathrm{~cm}^{-1}$ at $280 \mathrm{~nm}$ \\
\hline $\mathrm{A}_{280}$ & 0.903 \\
\hline $\mathrm{C}_{280}$ & 0.1806 \\
\hline$\varepsilon_{330 \text { reagent }}$ & $3690 \mathrm{M}^{-1} \mathrm{~cm}^{-1}$ at $330 \mathrm{~nm}$ \\
\hline
\end{tabular}

Table S2: Example data for degree of labeling calculation

$$
D O L=\frac{0.4711 * 43284 \mathrm{M}^{-1} \mathrm{~cm}^{-1}}{(0.903-0.4711 * 0.1806) * 3690 \mathrm{M}^{-1} \mathrm{~cm}^{-1}}=6.75
$$

Equation S2: Degree of labeling output for biotinylated BSA sample using equation S1.

\section{Degree of Labeling and HABA comparison.}

Bovine serum albumin was prepared at $0.15 \mathrm{mM}$ in $\mathrm{pH} 8.425 \mathrm{mM}$ bicarbonate solution at $25^{\circ} \mathrm{C}$. $100 \mu \mathrm{L}$ of the BSA solution was transferred to Eppendorf tubes, each labeling reaction was performed in triplicate. Immediately prior to reaction, $4 \mathbf{a}$ was prepared at $15 \mathrm{mM}$ in $\mathrm{DI}_{2} \mathrm{O} .30$ $\mu \mathrm{L}$ (30 eq) of the $\mathbf{4 a}$ solution was added to the BSA solution to produce to low DOL 4a-BSA conjugate and $150 \mu \mathrm{L}$ (150 eq) was added to produce the high DOL 4a-BSA conjugate. The solutions were vortexed immediately after addition and allowed to react for $1 \mathrm{~h}$ prior to the addition of $5 \mu \mathrm{L} \mathrm{NH} \mathrm{H}_{4} \mathrm{OH}$ to quench the reaction. $100 \mu \mathrm{L}$ of resulting solution was purified using a Princeton Separations Pro-Spin CS-800 gel permeation column, giving the final purified conjugate. The absorbance data was obtained using a Molecular Devices Spectramax instrument. HABA assays were performed using $120 \mu \mathrm{L}$ of HABA/avidin premix purchased from Sigma and adding $5 \mu \mathrm{L}$ of the low DOL purified conjugate or $2 \mu \mathrm{L}$ of the high DOL conjugate. The displacement was followed using absorbance at $500 \mathrm{~nm}$ for 30 minutes. The absorbance spectra 
of the $4 \mathbf{a}-\mathrm{BSA}$ conjugate was measured in a $500 \mu \mathrm{L}$ quartz cuvette by taking $40 \mu \mathrm{L}$ of the sample and diluting with $440 \mu \mathrm{L}$ of $25 \mathrm{mM} \mathrm{pH} 8.4$ bicarbonate solution.

\section{HRP Labeling and HABA determination}

Horseradish peroxidase was prepared at $0.15 \mathrm{mM}$ in $\mathrm{pH} 8.425 \mathrm{mM}$ bicarbonate solution at 25

${ }^{\circ} \mathrm{C} .100 \mu \mathrm{L}$ of the HRP solution was transferred to Eppendorf tubes, labeling reaction was

performed in triplicate. Immediately prior to reaction, 4a was prepared at $15 \mathrm{mM}$ in $\mathrm{DI} \mathrm{H}_{2} \mathrm{O} .30$

$\mu \mathrm{L}$ (30 eq) of the $\mathbf{4 a}$ solution was added to the HRP solution. The solutions were vortexed immediately after addition and allowed to react for $1 \mathrm{~h}$ prior to the addition of $5 \mu \mathrm{L} \mathrm{NH} \mathrm{NH}_{4} \mathrm{OH}$ quench the reaction. $100 \mu \mathrm{L}$ of resulting solution was purified using a Princeton Separations ProSpin CS-800 gel permeation column, giving the final purified conjugate. The absorbance and fluorescence data was obtained using a Molecular Devices Spectramax instrument. HABA assays were performed using $120 \mu \mathrm{L}$ of HABA/avidin premix purchased from Sigma and adding 14.1 $\mu \mathrm{L}$ of the purified conjugate. The displacement was followed using absorbance at $500 \mathrm{~nm}$ for 30 minutes. The absorbance spectra of the $\mathbf{4 a - H R P}$ conjugate was measured in a $500 \mu \mathrm{L}$ quartz cuvette by taking $40 \mu \mathrm{L}$ of the sample and diluting with $440 \mu \mathrm{L}$ of $25 \mathrm{mM} \mathrm{pH} 8.4$ bicarbonate solution. The fluorescence spectrum was obtained using an opaque 96 well plate. 4.1 $\mu \mathrm{L}$ of the $4 \mathrm{a}-\mathrm{HRP}$ conjugate was diluted to $41 \mu \mathrm{M}$ with $25 \mathrm{mM}$ bicarbonate solution for comparison to background fluorescence of unlabeled HRP. A calibration curve for $\mathbf{4 a}$ was prepared by reacting $4 \mathrm{a}$ with $\mathrm{n}$-butylamine. Excess butylamine was removed under reduced vacuum. This sample was then serially diluted with $25 \mathrm{mM}$ bicarbonate solution for use as a calibration standard for fluorescent experiments. 


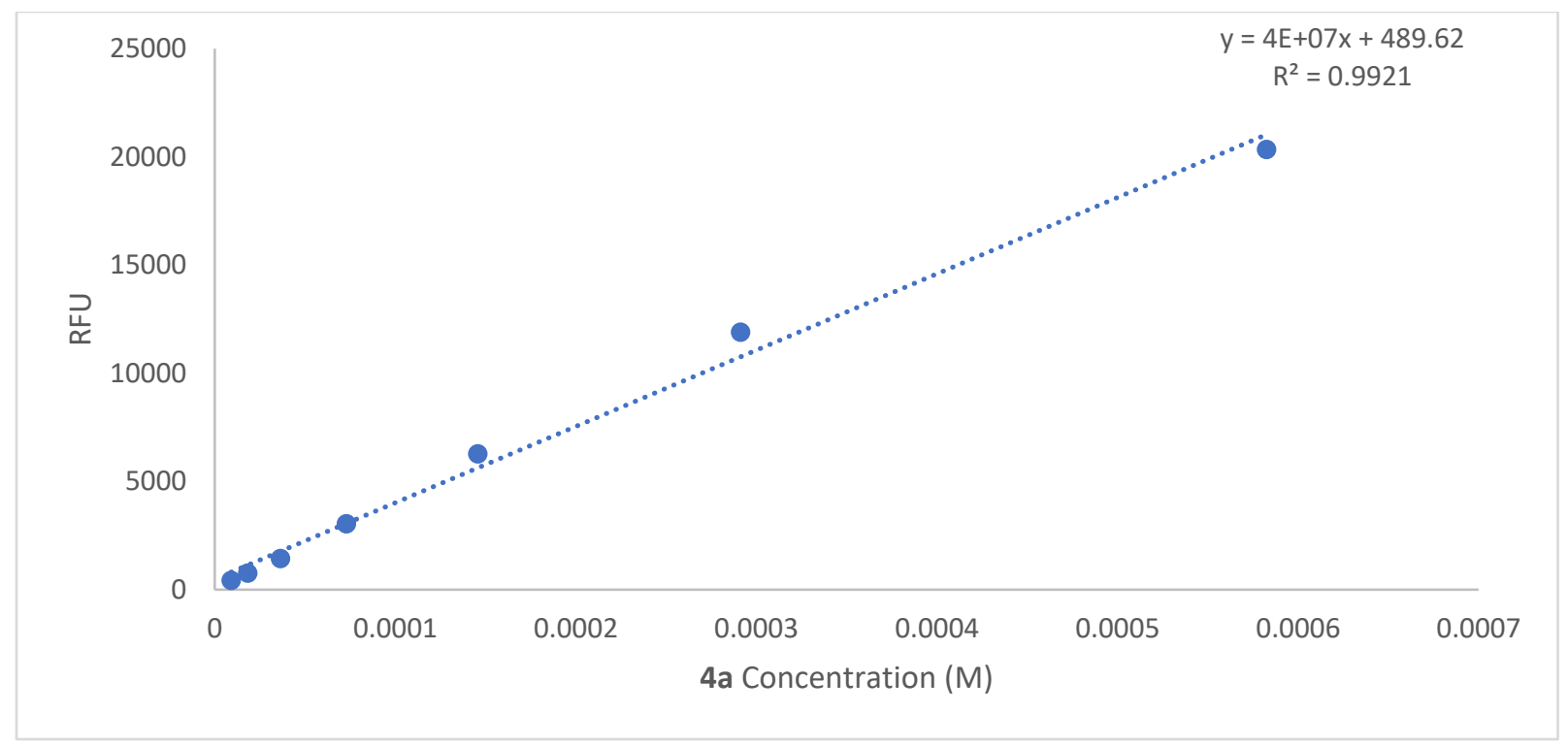

Figure S1: Calibration curve for $\lambda_{\mathrm{em}}$ at $420 \mathrm{~nm}$ of $\mathbf{4 a}, \lambda_{\mathrm{ex}}=330 \mathrm{~nm}$

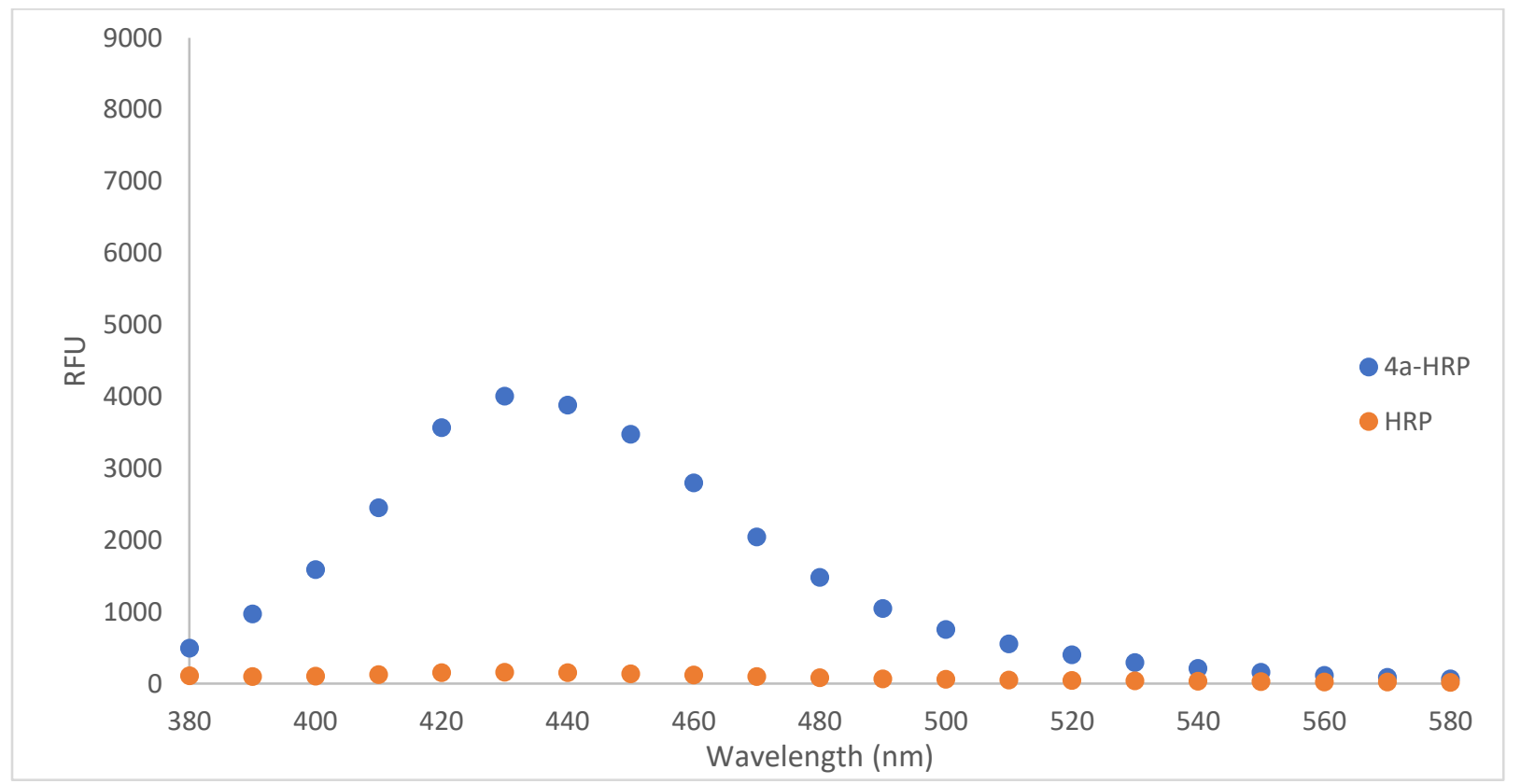

Figure S2: Fluorescence data for $\mathbf{4 a - H R P}$ conjugate vs unlabeled HRP at $41 \mu \mathrm{M}$ concentration in opaque 96 well plate 


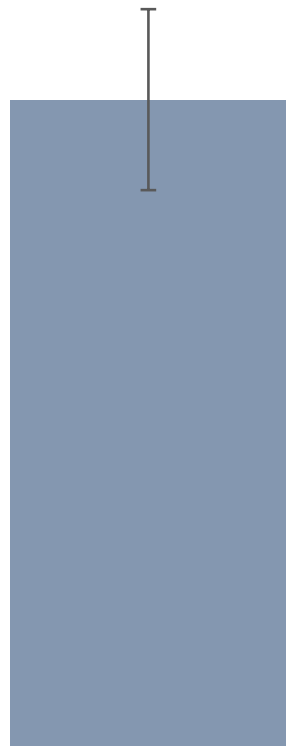

Figure S3: Unadjusted DOL data for 4a-HRP conjugate using HABA assay and fluorescence of 4a-HRP conjugate based on protein concentration and $\mathbf{4 a}$ fluorescence calibration curve

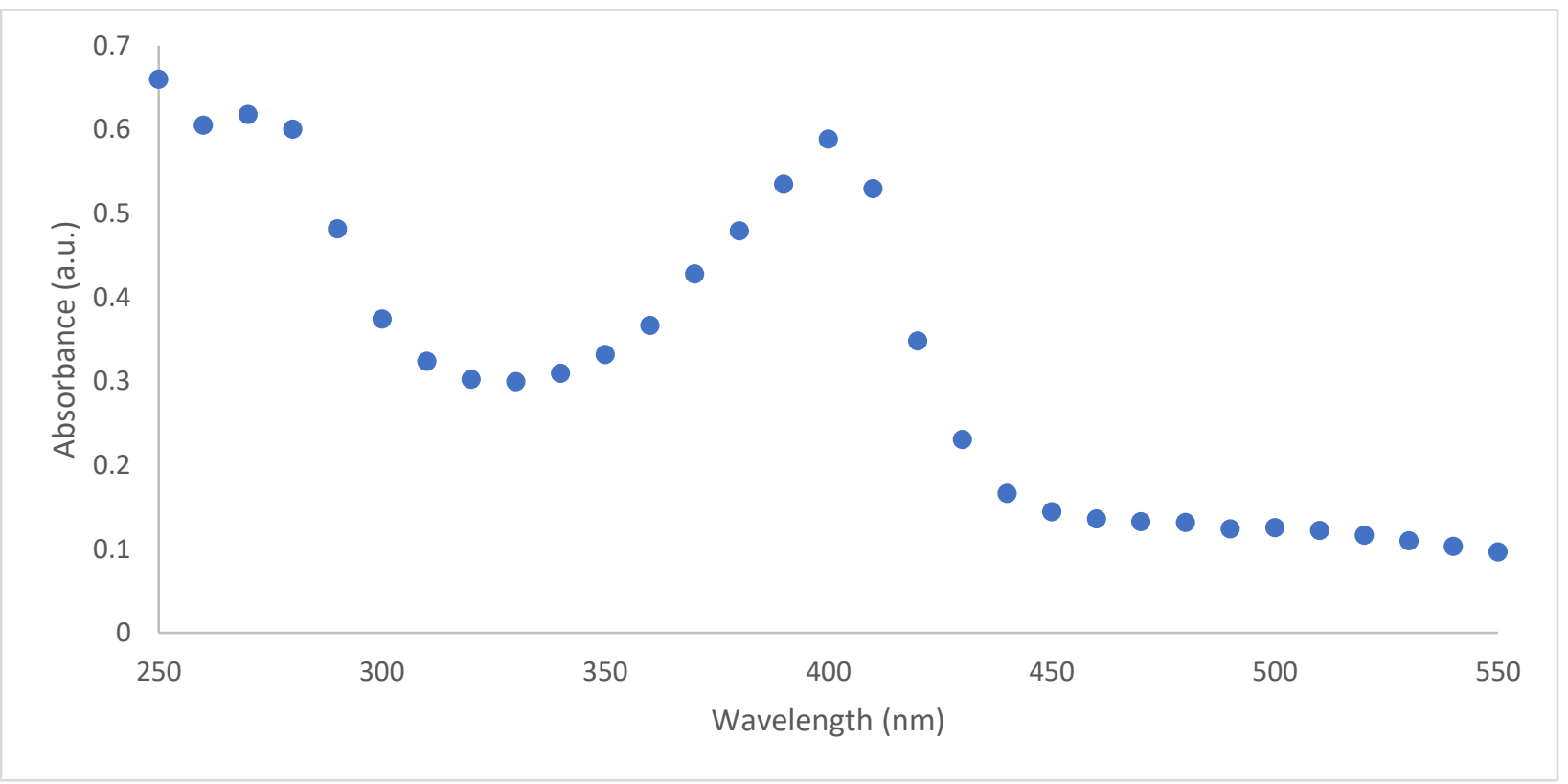

Figure S4: Broad absorbance spectra of HRP at $13 \mu \mathrm{M}$, replicating concentration as when used in HABA assay 


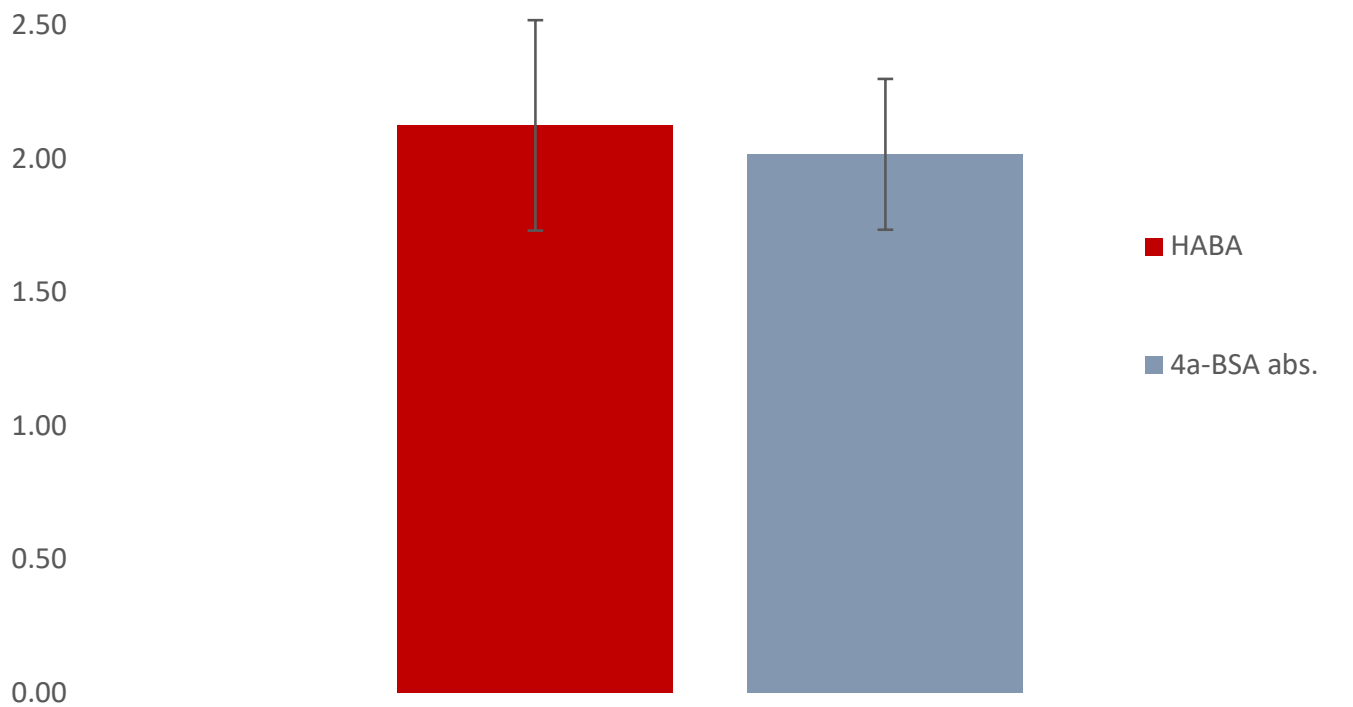

Figure S5: Adjusted DOL data by subtraction of $\mathbf{4 a - H R P}$ contribution to $500 \mathrm{~nm}$ absorbance of HABA assay

\section{Sulfo-NHS-biotin vs 4a labeling comparison.}

Comparison of $\mathbf{4 a}$ to sulfo-NHS biotin was done through labeling of BSA. $100 \mu \mathrm{L}$ BSA at 0.15 $\mathrm{mM}$ was placed in an Eppendorf tube, to this was added $45 \mu \mathrm{L}$ of labeling reagent (Sulfo-NHSbiotin or $4 \mathbf{a})$ at $10 \mathrm{mM}(30 \mathrm{eq})$. The reaction proceeded for $1 \mathrm{~h}$ then was quenched using $5 \mu \mathrm{L}$ $\mathrm{NH}_{4} \mathrm{OH}$. Samples were purified as detailed above with Princeton Separations Pro-Spin Cs-800. 5 $\mu \mathrm{L}$ of the sample mixture was used for $120 \mu \mathrm{L}$ of the HABA/avidin mix. Absorbance data was obtained using $40 \mu \mathrm{L}$ of sample was added to $440 \mu \mathrm{L} 25 \mathrm{mM}$ bicarbonate solution $\mathrm{pH} 8.4$ and placed in a $500 \mu \mathrm{L}$ quartz cuvette on a Molecular Devices Spectramax M5 plate reader. 


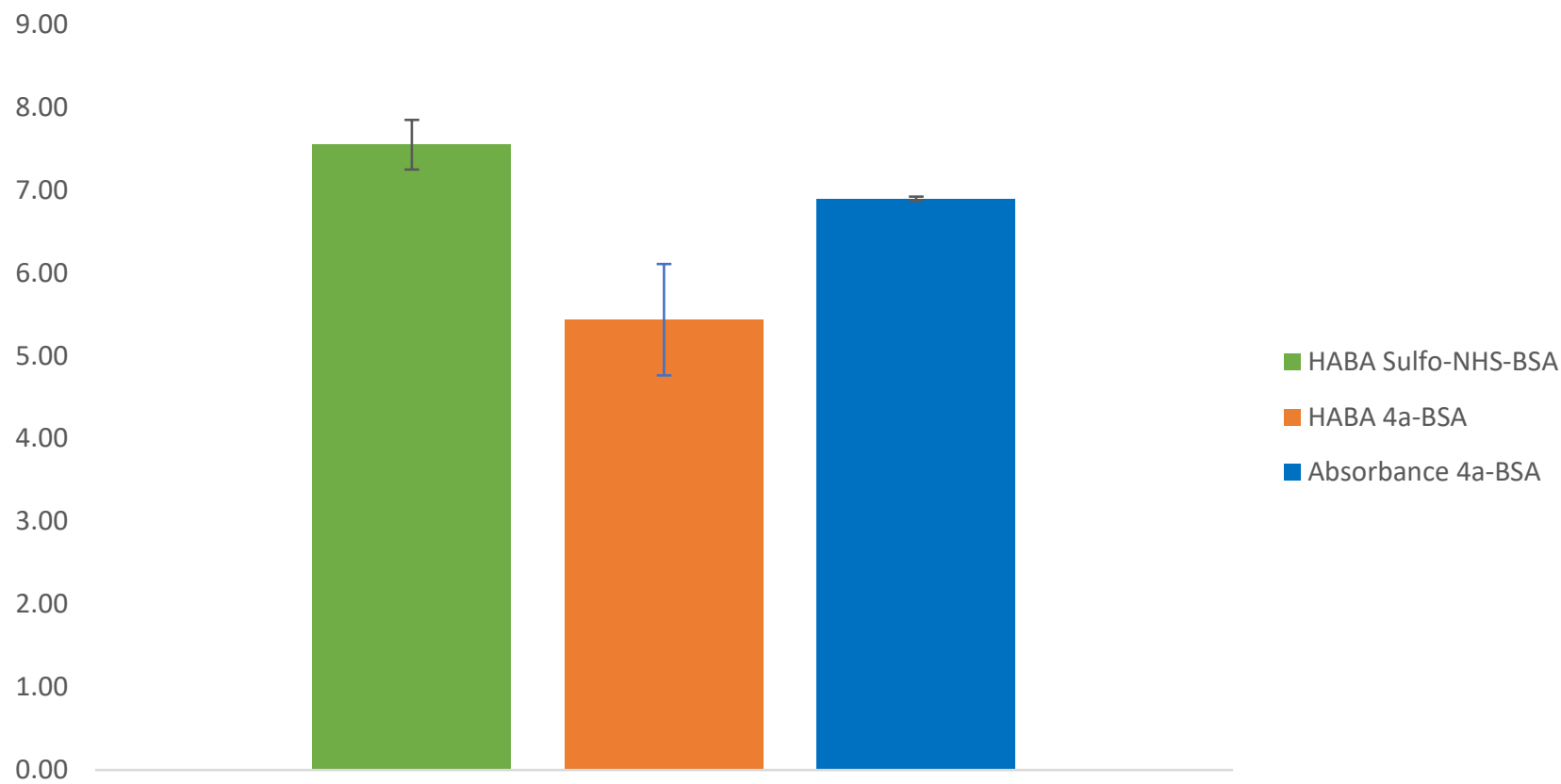

Figure S6: Comparison of Sulfo-NHS-biotin and 4a via HABA and absorbance data

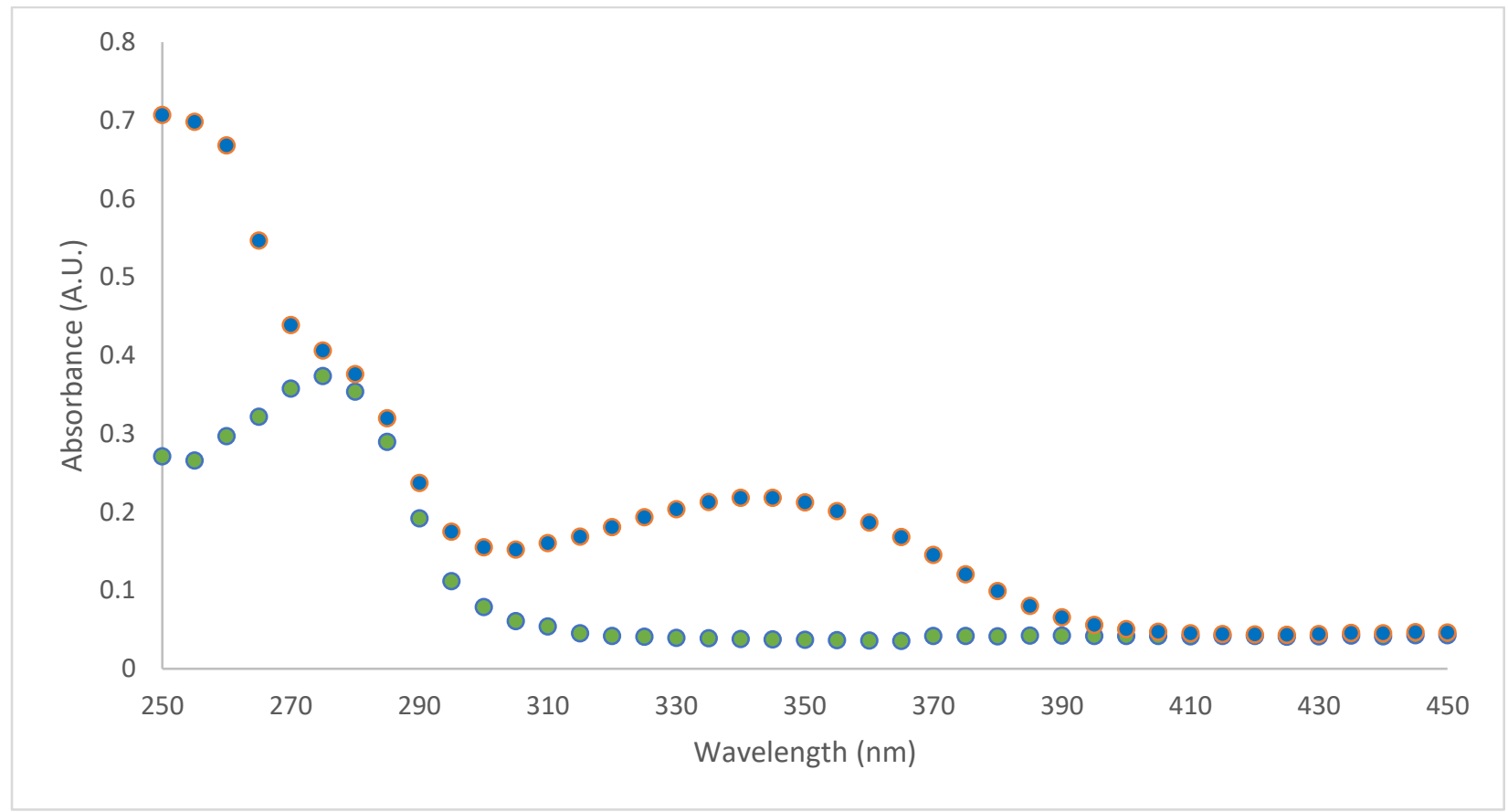

Figure S7: Absorbance data of 4a-BSA and Sulfo-NHS-biotin-BSA, $330 \mathrm{~nm}$ absorbance of isatoic anhydride readily seen at BSA concentration of $8.33 \mu \mathrm{M}$ 


\section{References:}

1. Fessler, A. B.; Dey, A.; Garmon, C. B.; Finis, D. S.; Saleh, N.-A.; Fowler, A. J.; Jones, D. S.; Chakrabarti, K.; Ogle, C. A., Water-Soluble Isatoic Anhydrides: A Platform for RNA-SHAPE Analysis and Protein Bioconjugation. Bioconjugate Chem 2018, 29 (9), 3196-3202.

2. Wang, T.-P.; Chiou, Y.-J.; Chen, Y.; Wang, E.-C.; Hwang, L.-C.; Chen, B.-H.; Chen, Y.-H.; Ko, C.H., Versatile Phosphoramidation Reactions for Nucleic Acid Conjugations with Peptides, Proteins, Chromophores, and Biotin Derivatives. Bioconjugate Chem 2010, 21 (9), 1642-1655. 